\title{
Cardiac rehabilitation outcome after transcatheter aortic valve implantation
}

\author{
Chiara Penati ${ }^{1}$, Cristoforo Incorvaia ${ }^{1}$, Valentina Mollo ${ }^{1}$, Federica Lietti ${ }^{1}$, Gemma Gatto ${ }^{1}$, \\ Marco Stefanelli ${ }^{1}$, Paola Centeleghe ${ }^{1}$, Giuseppe Talarico ${ }^{1}$, Ileana Mori ${ }^{1}$, Cristina Franzelli ${ }^{1}$, Fosco Ratti ${ }^{1}$, \\ Maria Paola Ponticelli ${ }^{1}$, Erminia Ridoloº ${ }^{2}$, Oreste Carlo Febo ${ }^{1}$ \\ ${ }^{1}$ Cardiac/Pulmonary Rehabilitation, ASST Pini-CTO Hospital, Milan; ${ }^{2}$ Allergy and Clinical Immunology, Medicine \\ and Surgery Department, University of Parma, Italy
}

\begin{abstract}
Patients with severe aortic stenosis are increasingly treated with transcatheter aortic valve implantation (TAVI) as a safer option to surgical aortic valve replacement (sAVR). Similar to many other heart diseases, after the specific therapeutic intervention patients are eligible for cardiac rehabilitation (CR) for the purpose of functional recovery. Thus far, CR after both sAVR and TAVI has been used to a limited extent, as shown by the availability of only two meta-analyses including 5 studies and 6 studies, respectively. Recent observational studies reported a significant improvement in functional indexes such as the Barthel scale and the 6-minute walk test (6MWT). We evaluated the outcome of CR in patients after TAVI treatment by measuring changes in the com-
\end{abstract}

\section{Correspondence: Cristoforo Incorvaia, Cardiac/Pulmonary Rehabilitation, ASST Pini-CTO, Milan, Italy. \\ E-mail: cristoforo.incorvaia@gmail.com}

Key words: severe aortic stenosis; transcatheter aortic valve implantation; cardiac rehabilitation; Barthel scale; 6-minute walk test; short physical performance battery scale.

Contributions: OCF, CI, CP, conception and design of the study, manuscript drafting; ER, data acquisition and analysis; GG, PC, methodology; VM, FL, MS, GT, IM, CF, FR, MPP, clinical evaluation and study procedures. All the authors have read and approved the final version of the manuscript and agreed to be accountable for all aspects of the work.

Conflict of interest: The authors declare no conflict of interest.

Guarantor: Oreste Carlo Febo, MD.

Ethics approval and consent to participate: No ethical committee approval was required for this study.

Received for publication: 24 September 2020.

Accepted for publication: 2 December 2020

${ }^{\circ}$ Copyright: the Author(s), 2021

Licensee PAGEPress, Italy

Monaldi Archives for Chest Disease 2021; 91:1621

doi: 10.4081/monaldi.2021.1621

This article is distributed under the terms of the Creative Commons Attribution Noncommercial License (by-nc 4.0) which permits any noncommercial use, distribution, and reproduction in any medium, provided the original author(s) and source are credited. monly used Barthel scale and 6MWT and adding the short physical performance battery (SPPB) scale as an index to assess lower extremity function. All indexes demonstrated a significant improvement, namely $\mathrm{p}<0.001$ with the Barthel scale, $\mathrm{p}=0.043$ for the $6 \mathrm{MWT}$, and $\mathrm{p}=0.002$ for SPPB. These results confirm the significant improvement of the Barthel scale and 6MWT reported in the previous meta-analysis and suggest the utility of SPPB as a further index of efficacy of CR in patients with severe aortic stenosis treated with TAVI.

\section{Introduction}

Severe aortic stenosis (AS) is burdened, if not treated, by significant mortality, which was estimated to occur in $36 \%$ of patients asymptomatic not referred for surgery, $73 \%$ of patients who are denied surgery due to comorbidities, and $18 \%$ in patients accepted for surgery [1]. In a recent survey on 241,303 patients with AS (mean age of $61 \pm 17$ years in males and $62 \pm 19$ years in females), the 5-year mortality in patients with moderate to severe AS (as defined by measured aortic valve mean gradient, peak velocity, and/or area), was $56 \%$ and $67 \%$, respectively [2]. Surgical aortic valve replacement (sAVR) has been performed since the 1960' [3] until transcatheter aortic valve implantation (TAVI) was introduced as a nonsurgical option free from the risks of conventional surgery. This resulted in a progressive increase of the number of patients in whom the valve implantation can be indicated [4,5]. The effectiveness of TAVI is supported by a Cochrane meta-analysis including 18 studies [6] and currently acknowledged in international consensus documents and guidelines [7,8]. As with other heart diseases, such as coronary artery disease, heart failure with reduced ejection fraction, and peripheral arterial disease, after the specific therapeutic intervention cardiac rehabilitation (CR) is aimed at restoring the previous level of functional capacity [9]. Based on the clear evidence from the literature, $\mathrm{CR}$ received a class $1 \mathrm{~A}$ recommendation by the American Heart Association/American College of Cardiology [10]. However, the number of studies addressing the outcome of CR in patients treated with TAVI is low. Actually, the two meta-analyses available thus far, including 5 studies and 6 studies, respectively, had conflicting efficacy, with significant improvement found by Ribeiro et al. [11] but not by Anayo et al. [12]. For these reasons, observational studies are important to evaluate characteristics, core components of intervention, and outcome in TAVI patients enrolled to CR.

The present study aimed at evaluating the outcome of CR in patients after TAVI treatment as assessed by changes in the Barthel scale, 6-minute walk test (6MWT), and short physical performance battery (SPPB) scale. 


\section{Patients and Methods}

The study considered 48 Caucasian patients following TAVI who underwent a residential $\mathrm{CR}$ program at the Complex Operational Unit of Cardiac/Pulmonary Rehabilitation of the ASST Pini-CTO Hospital between 01 January 2019 and 31 December 2019. Two patients were excluded because one resigned and the other was transferred due to complications. During hospitalization, all patients underwent training sessions six days a week: five days they performed 30-minute aerobic activities at low intensity (Borg 10/20) and 30 minutes group exercises alternating muscle strengthening (about 10 repetitions for 2 of 10 different exercises at $30 \%$ $50 \%$ of $1 \mathrm{RM}$ ) and stretching with balance exercises and coordination; on the sixth day they underwent only one of the two activities. Of the 46 patients included in the study, 19 were males and 27 were females, with an average age of 82.6 years in males and 81.2 in females. Tables 1 to 3 report patients characteristics according to nutritional status (Figure 1), therapy and comorbidities, respectively. Patients FE was not notably compromised upon arrival at our unit. At the entrance, 17 patients were walking with aid (16 with walker and 1 with stick), while 2 patients were bedridden; at discharge 5 of the 17 patients walked without aid, 9 maintained the aid used at the entrance, 3 went from the walker to the use of a stick and the 2 bedridden patients were able to walk with a walker. All patients were evaluated by the Barthel scale [13], half of them were assessed using the SPPB scale [14] and assigned to an aerobic training of deambulatory type with intermittent mode, alternating average repetitions of 250 meters with average breaks of $3 \mathrm{~min}$. The remaining patients were assessed by means of a 6MWT [15] and assigned to continuous aerobic training with the cycle ergometer. After 18 months from discharge, a follow-up was made by phone calls to evaluate the clinical status, hospitalizations and adherence to physical activity (Figure 2).

\section{Statistical methods}

The discrete variables, particularly the Chi-squared test if the populations in question included more than 10 units and the Fisher exact test or the Mann-Whitney test if they included less than 10 units, were used. The Student's $t$-test was used to test the association between continuous variables.

\section{Results}

Table 4 shows the data concerning patients age and the results of Barthel scale, 6MWT and SPPB before and after reha-
Table 1. Mean values of albumin, $\mathrm{Hb}$ and BMI.

\begin{tabular}{lcccc} 
& Albumin & Hb & BNI in & BVI out \\
Mean & 3.348478 & 10.01739 & 27.15234 & 26.9062 \\
Standard deviation & 0.299013 & 0.976457 & 5.075313 & 4.943296 \\
\hline Variance & 0.089409 & 0.953469 & 25.7588 & 24.43617 \\
Median & 3.34 & 9.85 & 26.61458 & 26.13814 \\
\hline Minimal value & 2.44 & 7.9 & 15.88697 & 17.23905 \\
Maximal value & 4.03 & 12.3 & 41.43705 & 41.00987 \\
\hline
\end{tabular}

Table 2. Therapy (number of patients under different kind of therapy).

\begin{tabular}{lc}
\hline Double anti-platelet agent & 25 \\
Single anti-platelet agent & 7 \\
\hline Anticoagulant + antiplatelet & 9 \\
Anticoagulant & 5 \\
\hline Beta blocker & 37 \\
Calcium antagonist & 20 \\
\hline Sartanic & 8 \\
ACE-inhibitor & 16 \\
\hline Anti-arrhythmic & 6 \\
Hypocholesterolemic & 32 \\
\hline Diuretic & 36 \\
Hypoclycemic & 25 \\
\hline Gastroprotectors & 46 \\
\hline
\end{tabular}

Table 3. Comorbidities.

\begin{tabular}{lcc}
\hline Diabetes & 14 & $30.43 \%$ \\
Hypercholesterolemia & 23 & $50.00 \%$ \\
\hline Hypertension & 39 & $84.78 \%$ \\
COPD & 8 & $17.39 \%$ \\
\hline Ischemic disease & 3 & $6.52 \%$ \\
Arteriopathy & 7 & $15.22 \%$ \\
\hline Kidney failure & 16 & $34.78 \%$ \\
Anemia & 3 & $6.52 \%$ \\
\hline Obesity & 3 & $6.52 \%$ \\
Vasculopathy & 3 & $6.52 \%$ \\
\hline
\end{tabular}

Table 4. Mean values of Barthel index, SPPB and 6MWT before and after CR.

\begin{tabular}{lcccccccc} 
& Age & Barthel in & Barthel out & SPPB in & SPPB out & 6VWT in & 6MWT out \\
Mean & 81.565 & 73.80435 & 90.21739 & 4.565217 & 7.043478 & 265.4348 & 327.1739 \\
Standard deviation & 5.260554 & 23.31392 & 16.53133 & 2.272885 & 3.052246 & 89.02136 & 111.2208 \\
\hline Variance & 27.67343 & 543.5386 & 273.285 & 5.166008 & 9.316206 & 7924.802 & 12370.06 \\
Median & 82 & 80 & 100 & 4 & 7 & 260 & 300 \\
\hline Minimal value & 64 & 15 & 40 & 0 & 2 & 120 & 180 \\
Maximal value & 95 & 100 & 100 & 10 & 12 & 430 & 660 \\
\hline
\end{tabular}


BMI

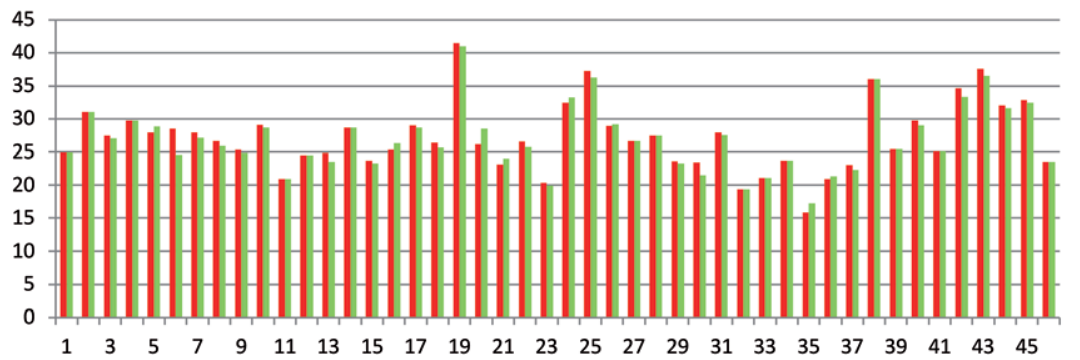

- BMl in $=\mathrm{BMl}$ out

BMI in

BMI out
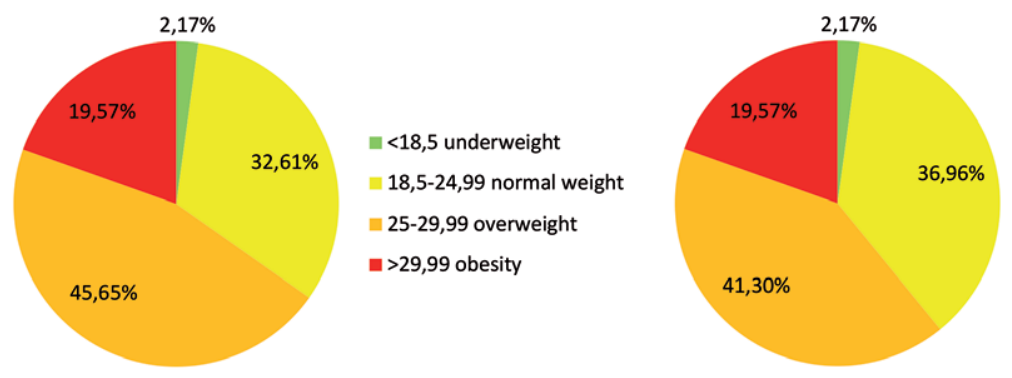

필 $<18,5$ underweight

118,5-24,99 normal weight

-25-29,99 overweight

$>29,99$ obesity

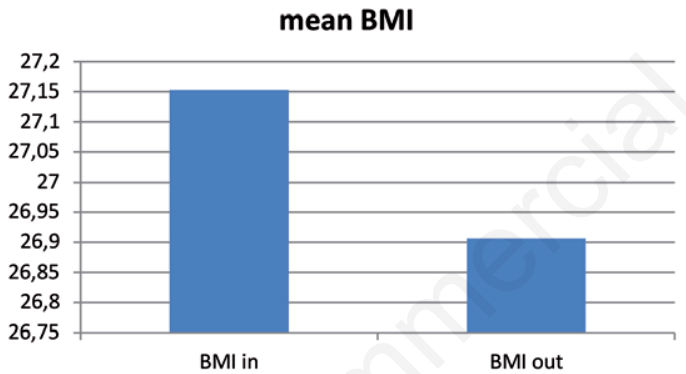

Albumin

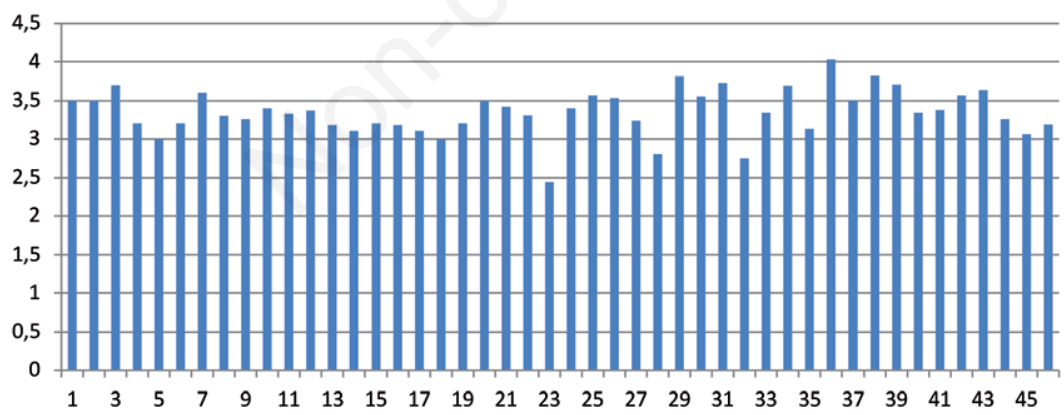

$\mathrm{Hb}$

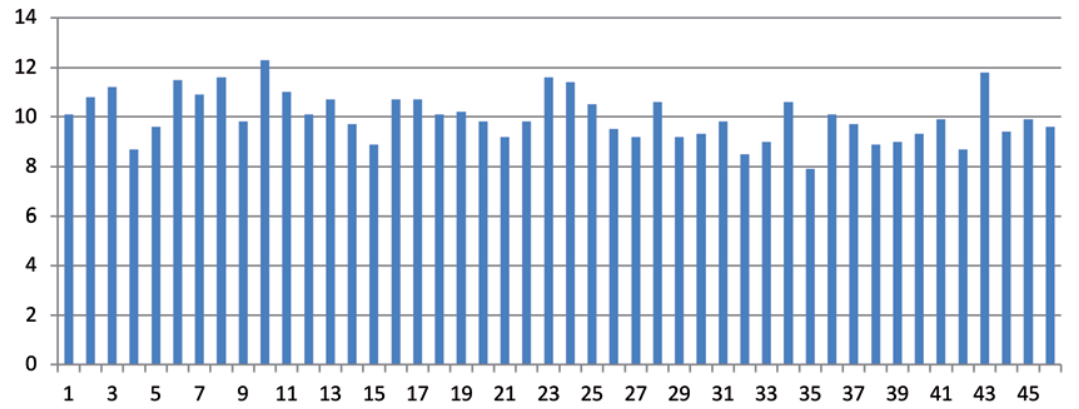

Figure 1. Nutritional status. 
STATE OF HEALTH

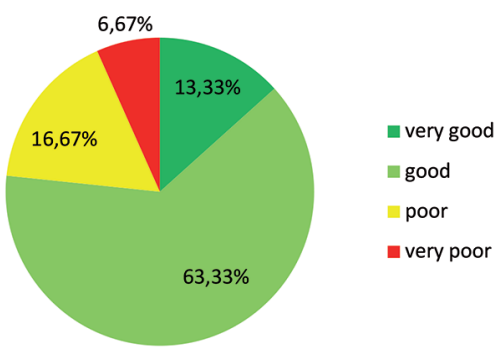

SUBSEQUENT HOSPITAL ADMISSIONS

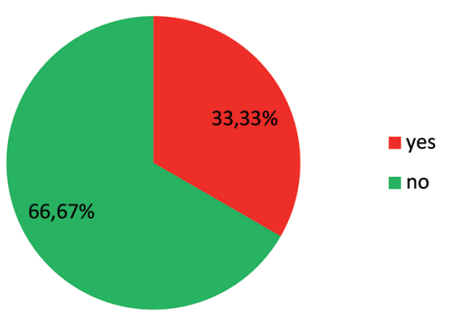

CAUSES OF SUBSEQUENT HOSPITAL ADMISSIONS

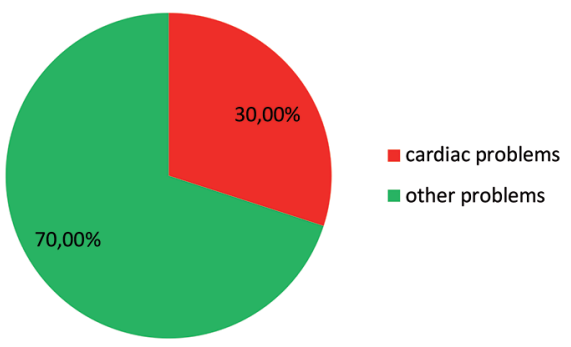

CONTINUING AEROBIC ACTIVITY**

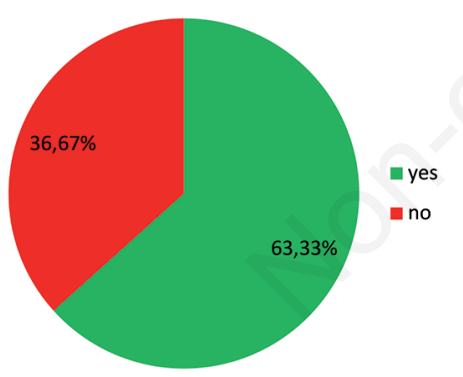

CONTINUING EXERCISES

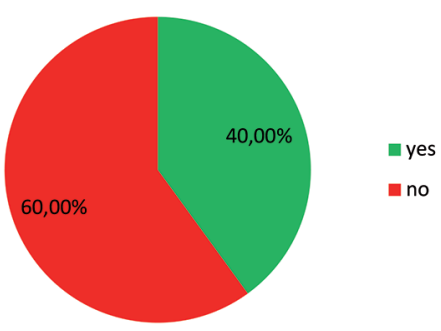

Figure 2. Follow up at 18 months. *Performed by telephone on 30 patients, as of 13 we did not have a telephone number and 3 did not answer; **aerobic activity performed in the year 2020, mostly at home or in the immediate vicinity, due to the COVID19 pandemic). bilitation. With the Barthel scale, the mean score was $73.80 \pm 23.31$ at entrance and $90.22 \pm 16.53$ at discharge $(p<0.001)$. In all patients there was an improvement in autonomy with the exception of patients who already had an input value of 100/100. The mean meters covered during 6MWT were $265.43 \pm 89$ in the initial test versus $327.17 \pm 111$ in the final test $(\mathrm{p}=0.043)$. The initial mean value of SPPB was $4.56 \pm 2.27$ versus the final meal value of $7.13 \pm 3.08(\mathrm{p}=0.002)$. Of the 23 patients assessed by SPPB, 7 also performed 6MWT at the entrance and exit. After an average of 3 weeks of rehabilitation, the rating scales of all patients showed an improvement in the score. In the 7 patients who performed both SPPB and 6MWT, a significant difference between the initial and final values was found for SPPB (from $6.4 \pm 1.9$ to $9.7 \pm 2.3$. $\mathrm{p}=0.013$ ), but not for $6 \mathrm{MWT}$. Figures 3 to 5 show the detailed changes in Barthel scale, SPPB and 6MWT before and after CR.

\section{Discussion}

After its introduction as a nonsurgical option to SAVR in patients with severe aortic stenosis, TAVI has been increasingly used [4,5]. A number of meta-analyses compared the outcomes of the two treatments. As to mortality, Carnero-Alcazar et al. analyzed 5 trials and 37 observational studies including 11,125 patients treated with sAVR and 9099 patients treated with TAVI, concluding that there was no significant difference in early or late mortality [16], while Panoulas et al. in a meta-analysis including 2052 males and 1706 females from 4 randomized controlled trials found that among females those treated with TAVI has a significantly lower mortality, as assessed by 26 to $35 \%$ lower mortality odds, than those treated with sAVR after 1 and 2 years [17].

As far as rehabilitation, regardless the kind of cardiac pathology, is concerned, the grade of scientific evidence from the literature resulted in a class $1 \mathrm{~A}$ recommendation by the American Heart Association/American College of Cardiology [10]. Still, despite such achievements, the number of studies addressing the outcome of CR is low, especially for patients treated with TAVI. In the first meta-analysis, which was based on 5 studies for an overall number of 292 patients treated with TAVI and 570 patients treated with sAVR, the CR program was associated with a significant improvement in 6MWT and in the Bartel index both for patients treated with TAVI and in those treated with sAVR $(\mathrm{P}<0.001$ for all measurements) [11]. Instead, in the meta-analysis by Anayo et al. including 6 studies (selected from the literature based on low risk of bias), with an overall number of 27 TAVI patients and 99 sAVR patients, the authors concluded that "exercise-based CR probably improves exercise capacity of post-TAVI and post-SAVR patients in the short term". The commonly used index to measure the improvement was exercise capacity as maximal oxygen uptake, while the 6MWT was used only in two studies [12]. It is apparent that the two meta-analysis differ in terms of inclusion criteria and efficacy indexes used.

As to observational studies, Eichler et al. enrolled 136 patients with elective TAVI undergoing CR to assess the effect on functional capacity, measured by 6MWT and maximum workload, and on quality of life measured by the Short Form-12 scale. Significant improvement was observed for both components [18]. Tarro Genta et al. evaluated the outcome of CR in 65 TAVI patients compared to 70 sAVR patients using 6MWT and Barthel index. Despite TAVI patients tolerated a significantly lower workload and had reduced 6MWT than sAVR patients, a net 
improvement in disability and exercise capacity was observed [19]. The results of our study, including only TAVI patients, confirm the significant improvement of the Barthel index and 6 MWT $(p<0.001$ and $p=0.043$, respectively) while adds SPPB (not evaluated in the meta-analyses) as a tool to assess frailty in patients undergoing CR [20], which was recently defined as a strong predictor for risk of physical disability in older adults entering CR [21]. The utility of specific tools assessing frailty for patients undergoing TAVI was highlighted by Giallauria and Vigorito, who suggested that "the adoption of a standardized and unique tool for assessing frailty in this cohort might help to identify specific rehabilitative pathways by tailoring the intervention

Barthel in Barthel out
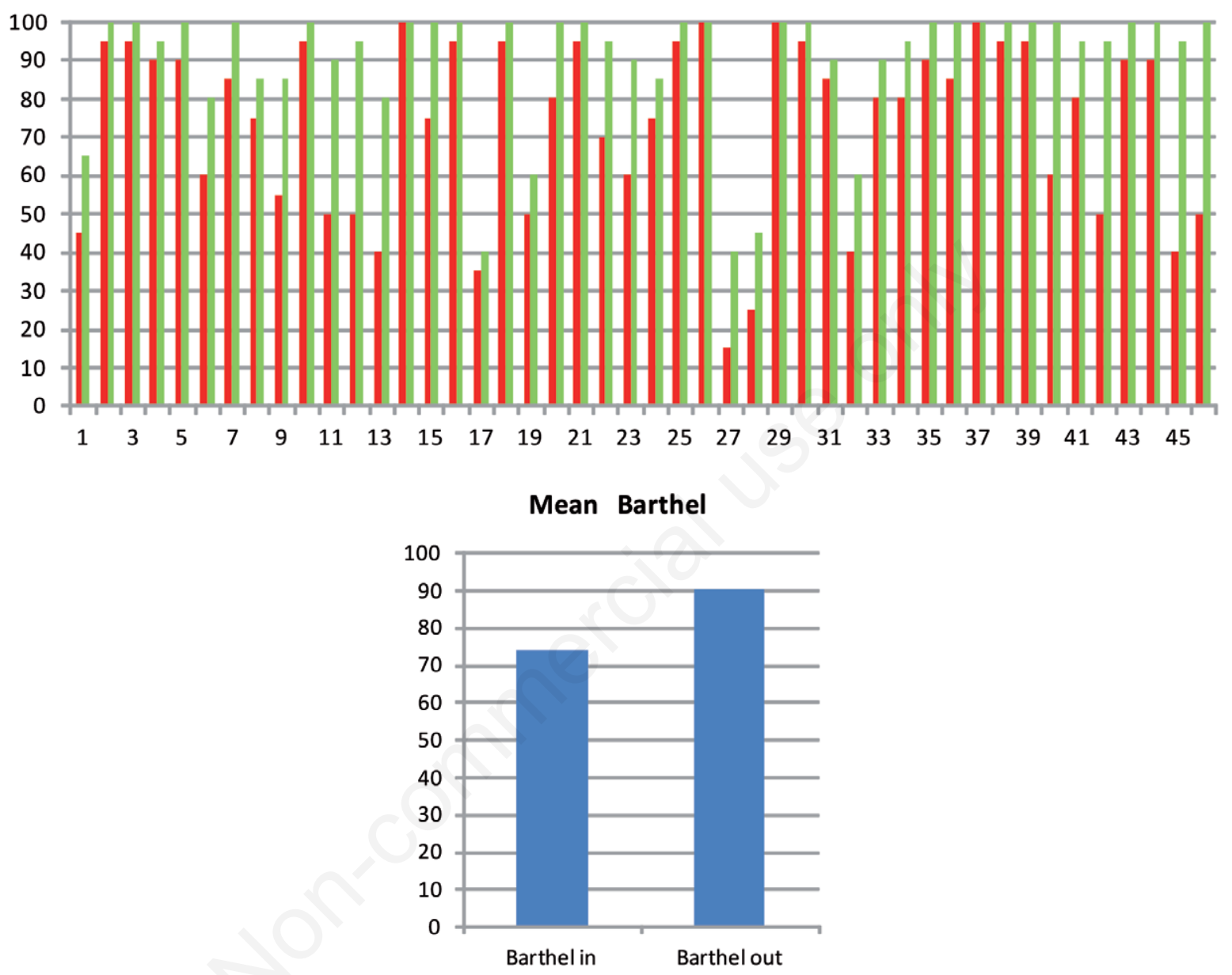

Barthel in

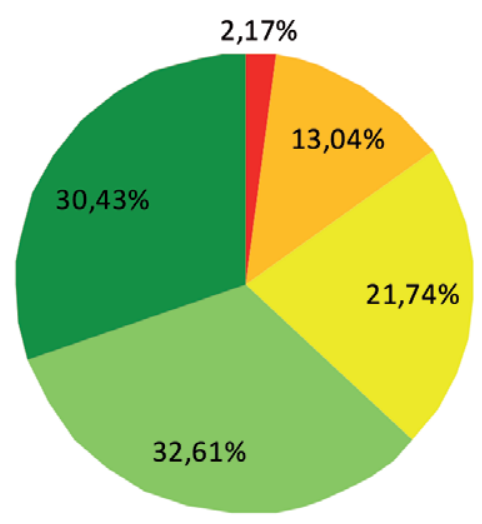

Barthel out

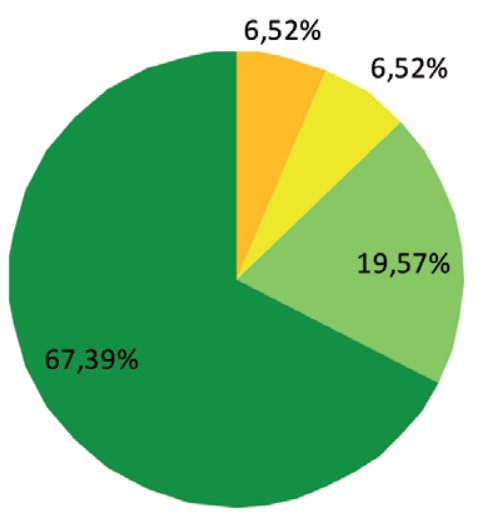

Figure 3. Detailed data from Barthel index at entrance and after cardiac rehabilitation. 
according to frailty scores" [22]. Furthermore, a recent multivariate regression analysis study on patients after mitral valve surgery showed that SPPB gave the best result in predicting unplanned readmission events [23]. Indeed, studies investigating the possible pathophysiological mechanisms underlying the dif- ferences observed between SPPB and 6MWT could improve the choices of functional indexes of physical capacity to be used. Based on our results, SPPB was significantly improved by CR, the initial mean value being $4.56 \pm 2.27$ while the mean value after CR was $7.13 \pm 3.08(\mathrm{p}=0.002)$. This suggests that adding SPPB to

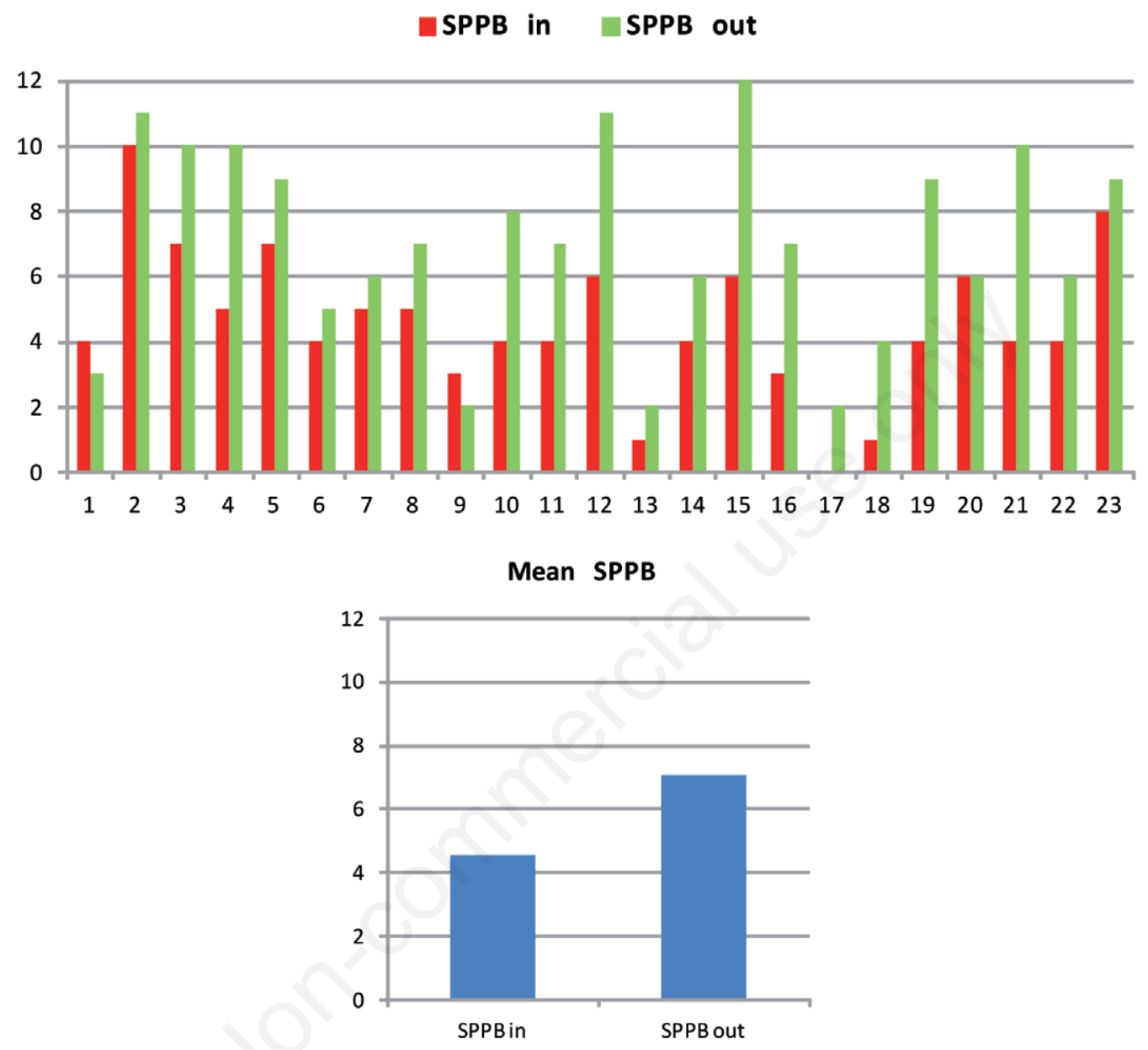

SPPB in

SPPB out

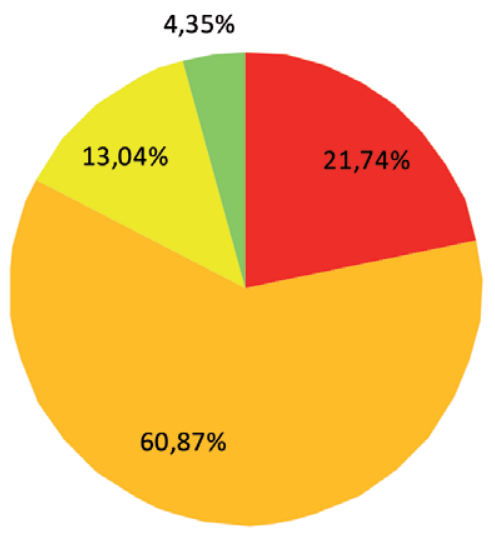

nsevere limitations

moderate limitations

mild limitations

minimal limitations

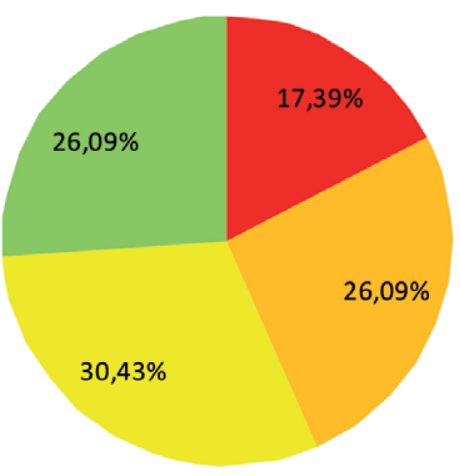

Figure 4. Detailed data from SPPB at entrance and after cardiac rehabilitation. 
the usual indexes for evaluating the success of $\mathrm{CR}$ in patients undergoing such treatment after TAVI may further improve the definition of the results obtained. The relatively small sample size and the observational nature of the study may have been limitation of the study.
In conclusion, the results of the present study confirm the significant improvement of the Barthel scale and 6MWT demonstrated in previous meta-analyses and indicate the usefulness of SPPB as an additional index in patients with severe aortic stenosis treated with TAVI.

6MWD in 6MWD out
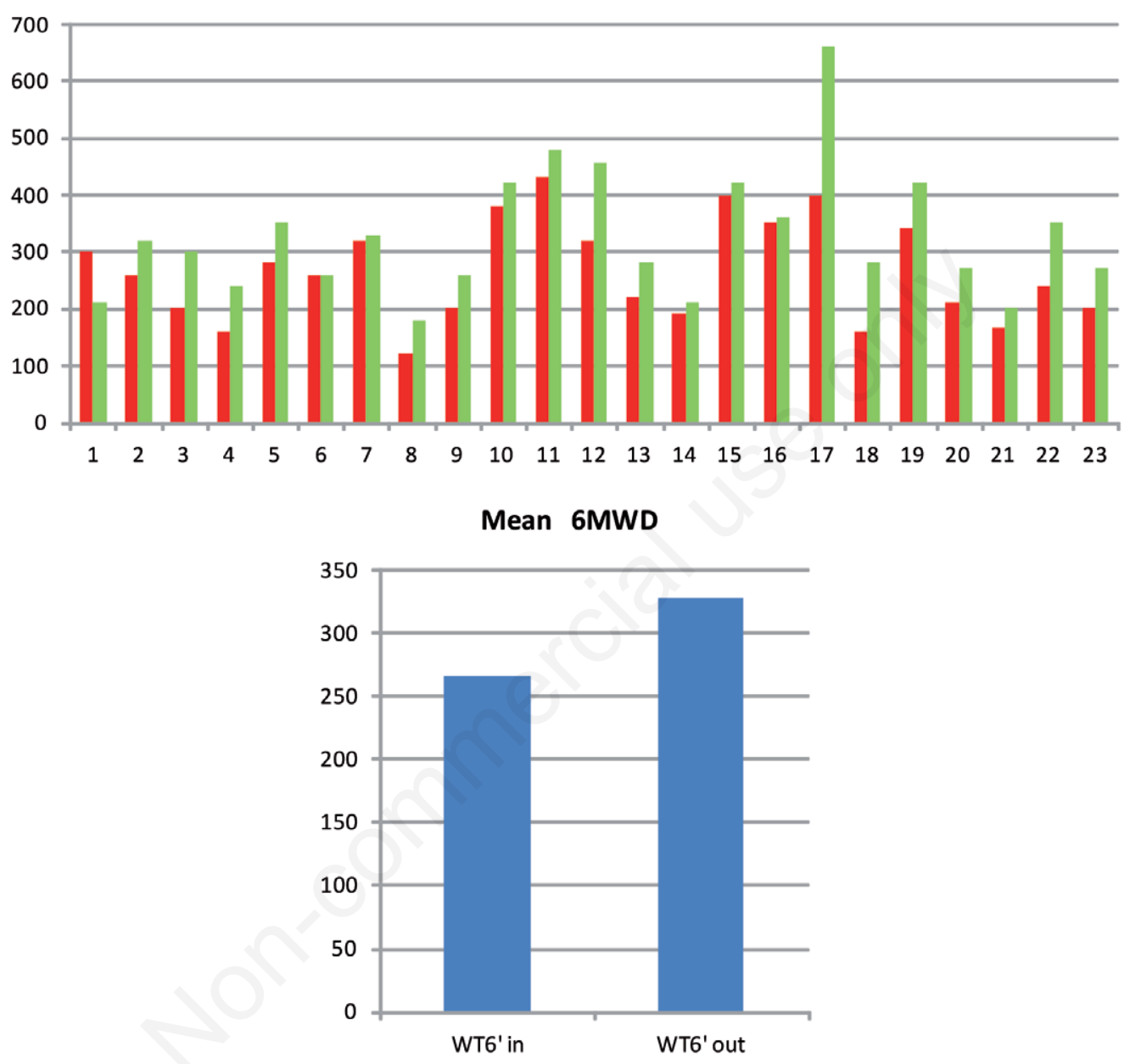

6MWD in

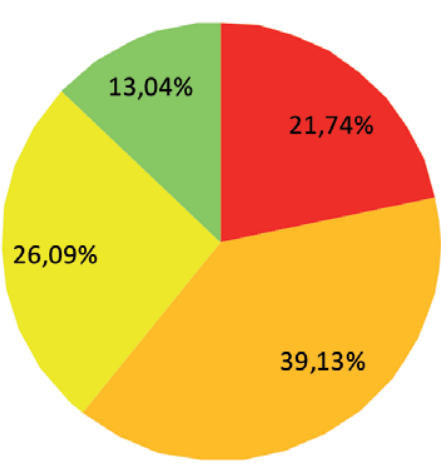

6MWD out

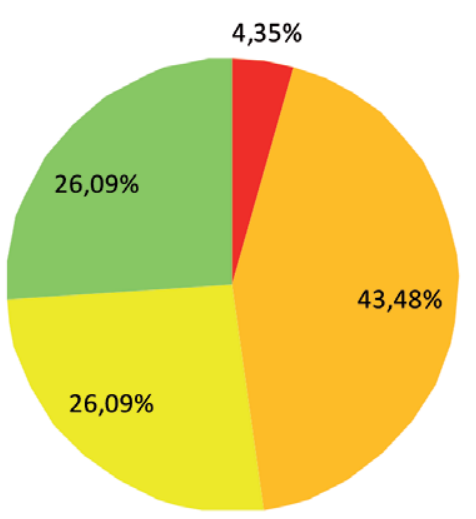

Figure 5. Detailed data from 6MWT at entrance and after cardiac rehabilitation. 


\section{References}

1. Perera S, Wijesinghe N, Ly E, et al. Outcome of patients with untreated severe aortic stenosis in real-world practice. N Zeal Med J 2011;124:40-8.

2. Strange G, Stewart S, Celermajer D, et al. Poor long-term survival in patients with moderate aortic stenosis. J Am Coll Cardiol 2019;74:1851-63.

3. Cribier AG. The odyssey of TAVR from concept to clinical reality. Tex Heart Inst J 2014;41:125-30.

4. Rostagno C. Heart valve disease in elderly. World J Cardiol 2019;11:71-83.

5. Randall MH, Bavry AA. Update on transcatheter aortic valve replacement. Cardiol Ther 2020;9:75-84.

6. Liu Z, Kidney E, Bem D, et al. Transcatheter aortic valve implantation for aortic stenosis in high surgical risk patients: a systematic review and meta-analysis. PLoS One 2018;13:e0196877.

7. Nishimura RA, Otto CM, Bonow RO, et al. 2017 AHA/ACC Focused Update of the 2014 AHA/ACC Guideline for the management of patients with valvular heart disease: a report of the American College of Cardiology/American Heart Association Task Force on Clinical Practice Guidelines. Circulation 2017;135:e1159-95.

8. Haude M. [Management of valvular heart disease: ESC/EACTS guidelines 2017].[Article in German]. Herz 2017;42:715-20.

9. Epstein E, Rosander A, Pazargadi A, et al. Cardiac rehab for functional improvement. Curr Heart Fail Rep 2020;17:161-70.

10. Thomas RJ, Balady G, Banka G, et al. 2018 ACC/AHA Clinical Performance and Quality Measures for Cardiac Rehabilitation: A report of the American College of Cardiology/American Heart Association Task Force on performance measures. Am Coll Cardiol 2018;71:1814-37.

11. Ribeiro GS, Melo RD, Deresz LF, et al. Cardiac Rehabilitation programme after transcatheter aortic valve implantation versus surgical aortic valve replacement: systematic review and metaanalysis. Eur J Prev Cardiol 2017;24:688-97.

12. Anayo L, Rogers P, Long L, et al. Exercise-based cardiac rehabilitation for patients following open surgical aortic valve replacement and transcatheter aortic valve implant: a systematic review and meta-analysis. Open Heart 2019;6:e00922.
13. Mahoney FI, Bartel DW. Functional evaluation: the Barthel index. Md State Med J 1965;14:61-5.

14. Guralnik, JM, Simonsick EM, Ferrucci L, et al. A short physical performance battery assessing lower extremity function: Association with self-reported disability and prediction of mortality and nursing home admission. J Gerontol 1994;49:M85-94.

15. Enright PL, McBurnie MA, Bittner V, et al. The 6-min walk test: A quick measure of functional status in elderly adults. Chest 2003;123:387-98.

16. Carnero-Alcazar, Maloto LM, Cobiella-Carnicer J, et al. Transcatheter versus surgical aortic valve replacement in moderate and high-risk patients: a meta-analysis. Eur Cardiothorac Surg 2017;51:644-52.

17. Panoulas VF, Francis DP, Ruparelia N, et al. Female-specific survival advantage from transcatheter aortic valve implantation over surgical aortic valve replacement: meta-analysis of the gender subgroups of randomized controlled trials including 3718 patients. Int J Cardio. 2018;250:66-72.

18. Eichler S, Salzwedel A, Reibis R, et al. Multicomponent cardiac rehabilitation in patients after transcatheter aortic valve implantation: Predictors of functional and psychocognitive recovery. Eur J Prev Cardiol 2017;24:257-64.

19. Tarro Genta F, Tidu M, Bouslenko Z, et al. Cardiac rehabilitation after transcatheter aortic valve implantation compared to patients after valve replacement. J Cardiovasc Med (Hagerstown). 2017;18:114-20.

20. Molino-Lova R, Pasquini G, Vannetti F, et al. Effects of a structured physical activity intervention on measures of physical performance in frail elderly patients after cardiac rehabilitation: a pilot study with 1-year follow-up. Intern Emerg Med 2013;8:581-9.

21. Rengo JL, Savage PD, Shaw JC, et al. Ades PA. Directly measured physical function in cardiac rehabilitation. J Cardiopulm Rehabil Prev 2017;37:175-181.

22. Giallauria F, Vigorito C. Editorial to 'Comparison of phase 2 cardiac rehabilitation outcomes between patients after transcatheter versus surgical aortic valve replacement'. Eur J Prev Cardiol 2018;25:1575-6.

23. Ashikaga K, Saji M, Takanashi S, et al. Physical performance as a predictor of midterm outcome after mitral valve surgery. Heart Vessels 2019;34:1665-73. 